\title{
Diferencias regionales y despliegue de Gobierno Electrónico en las provincias argentinas*
}

\author{
Vaca, J osefina** \\ Cao, Horacio $* * *$ \\ ** Licenciada en Educación. Investigadora de la Universidad Nacional de Quilmes. \\ Cursante de la Maestría en Ciencia Tecnología y Sociedad (UNQ). Email: \\ jvaca@fibertel.com.ar \\ *** Licenciado en Ciencia Política, Doctor en Administración (UBA). Investigador del \\ CIAP. Facultad de Ciencias Económicas-Universidad de Buenos Aires (UBA). Email: \\ hcao@fibertel.com.ar
}

\section{Resumen}

Este trabajo presenta algunos resultados obtenidos en una investigación desarrollada en el ámbito del Consejo Federal de Inversiones (CFI) de la República Argentina. Específicamente, y aunque en dicha investigación se analizaron diferentes aspectos del despliegue de las Tecnologías de Información y Comunicación en las provincias, en este artículo, presentaremos exclusivamente aquellos vinculados al desarrollo del gobierno electrónico. Se trata de una tarea mixta, realizada en parte sobre fuentes secundarias y en parte sobre tarea de campo propia. Muchos de los datos que se presentan son absolutamente originales ya que no se conocen antecedentes de recopilación de este tipo de información con un nivel de desagregación provincial y regional. Los resultados obtenidos ofrecen una primera mirada sobre el nivel de desarrollo del e-gov en las distintas jurisdicciones provinciales, el que parece guardar una estrecha relación con la heterogeneidad y desigualdad territorial del país.

Palabras clave: Provincias, gobierno electrónico, tecnologías de información y comunicación.

\section{Regional Differences and the Development of Electronic Government in Argentine Provinces}

\begin{abstract}
The objective of this paper is to present some of the results of a research project
\end{abstract}


developed within the scope of the Federal Council of Investments (CFI) in the Argentine Republic. Although in this research different aspects were analyzed as to the unfolding of the Information and Communication Technologies in the provinces, in this article, we refer exclusively those related to the development of electronic government. It is a mixed study based on secondary sources and original field study. Much of the information is absolutely original: and there are no known antecedents for this type of information with provincial desegregation. The information about the development of e-gov in the different provincial jurisdictions shows a close relationship with the heterogeneity and territorial inequality of the country.

Key words: Argentina, province, electronic government, informational and communicational technologies.

Recibido: 03-08-08. Aceptado: 03-10-03

\section{I ntroducción}

El propósito de este trabajo es el de realizar una primera descripción de las características del despliegue del Gobierno Electrónico en las provincias de la República Argentina. Esta visión es relevante en virtud de la complementación de dos factores:

- El alto grado de desigualdad y heterogeneidad regional del país, habida cuenta de la existencia hacia su interior de diferentes patrones sociales y especializaciones productivas por un lado y grados relativos de desarrollo y modernización, por otro.

- La creciente importancia que adquiere el fenómeno de gobierno electrónico, en tanto en su desarrollo se juegan elementos tales como la posibilidad de democratizar el funcionamiento del Estado, facilitar el acceso del conjunto de la sociedad a la revolución informacional, rediseñar las relaciones entre el Estado y la Sociedad, etc.

Nuestra aproximación al análisis del gobierno electrónico se realiza a partir de una tarea de campo efectuada en cada una de las provincias de nuestro país. Este relevamiento fue diseñado sobre la base de indicadores seleccionados en virtud de su pertinencia para observar el objeto bajo análisis, de su disponibilidad o posibilidad de construcción ad hoc y de su capacidad de cumplir con atributos básicos de la información tales como pertinencia, confiabilidad, claridad, etc. Cabe acotar que los datos existentes antes de realizar esta investigación eran muy escasos, situación originada en lo reciente del fenómeno e-gov. 
En cuanto a la presentación de la información, y tomando en cuenta que nuestra visión prioriza el factor regional, los datos se transcriben por provincias y por grupos de provincias. En este último caso, se sigue un agrupamiento que, en función de las características socioeconómicas provinciales, realiza el Banco Mundial. En la Tabla 1 se presenta dicha clasificación.

Esta forma de presentar la información permite observar con mayor claridad la correspondencia - o no- entre las características más estructurales de la sociedad y el nivel de desarrollo del e-gov. En este sentido, como hipótesis a priori, podría esperarse que en el caso del gobierno electrónico se reproduzca lo que se conoce como el "principio de equiparación" (Dhal, 1983: 84). Este principio sostiene que aunque pueden encontrarse excepciones, una región que se caracteriza por un cierto desarrollo relativo en un factor o dimensión, muy probablemente tendrá ese mismo nivel de desarrollo relativo en otros factores. Así, es de esperar que las provincias de mayor rezago relativo tengan una situación equivalente en lo que respecta al progreso del gobierno electrónico en su territorio.

\section{Criterios generales para la recopilación de la información}

La primera tarea fue la de seleccionar los indicadores a recopilar. Como punto de partida teníamos un conjunto de 105 indicadores incluidos en lo que se denominó "Propuesta Estándar de Medición de las TICS" (PEM), construido a partir de una investigación auxiliar1. La PEM, desarrollada sobre la base de experiencias nacionales e internacionales en el tema, contiene los elementos que se consideraron, no podían obviarse en ocasión de realizar un seguimiento permanente e integral de la situación de las tecnologías infocomunicacionales en cada una de las jurisdicciones. Sobre una porción de estos indicadores se realizó la tarea de campo que se transcribe.

Un primer rastreo, mostró que la oferta de datos homogéneos y consistentes disponibles era muy escasa, observando además, significativas diferencias en la calidad de la información provincia por provincia. Como ya adelantamos, el hecho de que las TICs sólo recientemente pasaran a ocupar un lugar en la agenda pública hace que no se hayan generado aún los mecanismos para que, al menos sus aspectos más importantes, sean captados de manera sistemática por las diferentes unidades públicas encargadas de relevar información. 
Tabla 1

Argentina valorización de popularidad Escalas para Poder Ejecutivo, Legislativo y Judicial

\begin{tabular}{lccc}
\hline & \multicolumn{3}{c}{ Puntos asignados } \\
\cline { 2 - 4 } Poderes & Baja & Media & Alta \\
\hline Poder Ejecutivo & Menor a 100 & de 101 a 1.000 & más de 1000 \\
Poder Legislativo & Menor a 10 & de 11 a 50 & más de 50 \\
Poder Judicial & Menor a 10 & de 11 a 20 & más de 20 \\
\hline
\end{tabular}

Fuente: Elaboración propia.

En resumen, estos factores llevaron a que una parte muy importante de la información se recavara a través de tareas de campo diseñadas y coordinadas por el equipo de investigación, las que aportaron la mayor parte de los insumos de la medición. Asimismo, fue crucial contar con la Red de Información y Conectividad del Consejo Federal de Inversiones ( $\mathrm{CFI})$, cuya colaboración permitió recolectar datos referidos al avance del gobierno en línea y de las regulaciones locales respecto de las TICs en cada jurisdicción. Otra faceta de la tarea de campo se llevó a cabo a través de Internet mediante una investigación sobre los sitios oficiales de cada jurisdicción. Para esto se trabajo con una metodología concebida para que los resultados fueran totalmente comparables.

- Los datos se recopilaron y trabajaron de dos maneras:

- Diferenciando cada una de las 24 jurisdicciones provinciales.

Siguiendo una clasificación que agrupa a las provincias en virtud de su desarrollo socioeconómico 2 , con el objetivo de observar en qué medida este desarrollo se articulaba con el nivel de despliegue del e-gov.

\section{Metodología utilizada}

\subsection{Aspectos generales}

Se conoce como "e-gobierno", "e-gov", "gobierno en línea" o "gobierno electrónico" a la utilización de nuevas tecnologías de información y comunicación (TICS) en el ámbito del Estado y de la Administración Pública. Esquemáticamente, puede decirse que la necesidad de incorporar estas tecnologías a los diferentes ámbitos del Estado se ven espoleadas a partir de:

- La manera de reconstruir capacidad de regulación del Estado sobre los diferentes flujos que se despliegan en la sociedad. En este aspecto, cabe resaltar que debe existir algún tipo de simetría entre el desarrollo tecnológico con el que cuente el 
organismo regulador y el organismo regulado para que el primero pueda cumplir sus funciones.

- La exigencia de responder a las crecientes demandas de la sociedad paralela a las dificultades para incrementar el nivel de recursos fiscales. En este caso la incorporación de las TICs está relacionada con la necesidad de un significativo incremento de la productividad para cerrar la brecha que se produce por la distancia entre objetivos y recursos.

- La necesidad de la ciudadanía de tener una relación más directa y transparente con los procesos administrativos que se desarrolla en el Sector Público, como así también por establecer canales directos de comunicación y control hacia la esfera política.

En la Argentina existen escasos antecedentes de tareas de campo dirigidas a obtener información acerca del despliegue del "e-gobierno" y poca precisión acerca de cuáles son los indicadores clave para dar señales sobre su situación global.

Esta situación, unida al carácter complejo y difícil de sintetizar en pocos registros del egov, nos llevó a optar por construir una batería de indicadores en nuestro objetivo de captar sus principales dimensiones. Así, se trabajó con ocho variables compuestas cada una de ellas por varias dimensiones, alcanzando un total de 27 subvariables $\underline{3}$, las que hacen referencia a diferentes aspectos del gobierno electrónico en cada uno de los tres poderes.

Como podrá advertirse en la próxima sección, las diferentes dimensiones fueron diseñadas bajo un perfil binario, asignándose un punto por cada aspecto considerado positivo y penalizándose con cero punto la ausencia de dicho aspecto. Las excepciones a esta regla se encuentran en la medición del nivel de actualización del sitio web, que va desde tres puntos positivos en los casos de actualización on line hasta un punto negativo si se observa más de un mes de desactualización, y en los casos en que existen regulaciones locales que afecten a las TICS, variable de característica inversa cuya presencia también asigna un punto negativo.

Como consecuencia de este diseño, cada jurisdicción termina teniendo asignado un puntaje, el que tiene un valor que va desde los dos (2) puntos negativos hasta los veintiocho (28) puntos positivos.

\subsection{Indicadores de e-gobierno}


a. Disponibilidad del gobierno en línea para los ciudadanos

El grado de disponibilidad de estos recursos permite ver en que medida los gobiernos están incorporando las TICs a sus procesos y productos organizacionales como así también a generar otro tipo de ámbito de relación con los ciudadanos.

- Existe algún sitio web del Gobierno provincial:

- con información en línea a disposición de los usuarios > + 1 punto

- que ofrezca servicios y trámites en línea a los ciudadanos $>+1$ punto

- que posibilite la contratación en línea $>+1$ punto

b. Normativas

La existencia de estas normativas permite observar, de que manera los gobiernos están marcando las pautas dentro de las que desarrollará este fenómeno hacia la propia organización. Pudiendo, además, medir la existencia de marcos jurídicos y políticas tendientes a conducir el cambio o simplemente a la adaptación de sus efectos.

- Existe algún marco normativo que reglamenta los sitios web de los entes públicos provinciales $>+1$ punto

- Existe alguna directiva interna del gobierno provincial para incorporarse a la web > +1 punto

- Existe alguna estrategia o plan maestro respecto del gobierno en línea $>+1$ punto

- Existe algún organismo que centralice las políticas de TICs $>+1$ punto

c. Disponibilidad del gobierno en línea para contratistas y proveedores

Con este grupo de indicadores se busca saber si los gobiernos provinciales están aprovechando las TICs para optimizar sus relaciones con contratistas y proveedores.

- El gobierno publica las compras ya realizadas $>+1$ punto

- El gobierno publica las compras que están siendo licitadas $>+1$ punto

- Las gestiones del gobierno y sus interacciones con contratistas y proveedores ocurren totalmente en línea $>+1$ punto

d. Regulaciones locales que afectan a las TICS

Estos datos permiten observar si existen elementos regulatorios dentro del gobierno que estén operando sobre el desarrollo de las TICs mediante la intervención en el mercado, generando un indicador por aproximación de políticas públicas sobre TICs.

- Existen impuestos locales específicos sobre las TICs > (-)1 punto 
- Existen entes del Estado provincial y/o regulaciones que favorecen el desarrollo de las TICs $>+1$ punto.

e. Políticas locales de estímulo del uso y desarrollo de las TICs

Estos datos nos permiten observar si existe algún elemento de promoción que favorezca el despliegue de las TICs mediante la intervención del Gobierno en el mercado, siendo al igual que el anterior un indicador por aproximación de políticas públicas sobre TICs.

- Existen regímenes de promoción sectorial a las empresas de las TICS $>+1$ punto.

- Existe algún régimen provincial de incentivo o promoción a la inversión en TIC por parte de las empresas $>+1$ punto.

f. Evaluación de los sitios web oficiales

El hecho de que cada uno de los tres poderes de los gobiernos provinciales poseyeran un sitio en la red es de por sí un indicador importante, pero se ha considerado conveniente analizarlos a la luz de la calidad de su funcionamiento. La valorización de los aspectos seleccionados genera una idea clara y comparable del sitio en términos de accesibilidad y actualización.

\section{Sitio web del Poder Ejecutivo Provincial}

Tiene sitio web $>+1$ punto

Accesibilidad $\stackrel{4}{>}+1$ punto

- Acceso

- Velocidad

- Compatibilidad con los buscadores

- Popularidad del sitio

- Actualización

- On line: por los organismos de la Red y los usuarios $>+3$ puntos

- Diaria: una vez al día por un responsable del sitio $>+2$ puntos

- Semanal: una vez a la semana por un responsable del sitio $>+1$ punto

- Mensual: una vez al mes por un responsable del sitio > 0 punto

- Desactualizada: más de un mes por un responsable del sitio > (-)1 punto

Sitio web del Poder Legislativo Provincial

Algunas provincias tienen una organización bicameral y, consecuentemente, cada cámara cuenta con un sitio web. En este caso se considera el promedio simple de los resultados. 
- Tiene sitio web $>+1$ punto

- Accesibilidad (ídem poder ejecutivo - ver Metodología)

- Contenido

- Información general: cámaras, bloques, comisiones, reglamentos internos $>+1$ punto

- Bases de datos: proyectos, legislación, versiones taquigráficas $>+1$ punto

- Compras y licitaciones $>+1$ punto

\section{$\underline{\text { Sitio web del Poder Judicial de la Provincia }}$}

- Tiene sitio web $>+1$ punto

- Accesibilidad (ídem poder ejecutivo- ver Metodología)

- Contenido

- Gestión de la oficina judicial: registro y seguimiento de causas $>+1$ punto

- Grandes registros: de peritos, de abogados, público de comercio $>+1$ punto

- Bases de datos documentales: jurisprudencia, dictámenes, acordadas, leyes, decretos, doctrina $>+1$ punto

- Compras y licitaciones $>+1$ punto

\section{Los resultados del relevamiento}

Como marco general del análisis, cabe acotar que algunos estudios ubican a la Argentina en una muy buena posición en el ámbito internacional en cuanto a gobierno electrónico. Según el World Markets Research (2001) ocupa el puesto № 19 entre 196 países y según el Programa de Administración Pública de las Naciones Unidas se encuentra un 50\% por encima del promedio mundial, ocupando la categoría de "alto nivel de egov" (ONU, 2003).

Ahora bien, al analizar la situación hacia adentro del territorio nacional el gobierno electrónico muestra en las distintas jurisdicciones y áreas, diferentes niveles de desarrollo. En una primera mirada, estas diferencias reproducen el patrón socioeconómico de la clasificación de provincias utilizada en la investigación ${ }^{\underline{5}}$. La delimitación provincial se detalla en el Mapa 1. 


\section{Mapa 1}

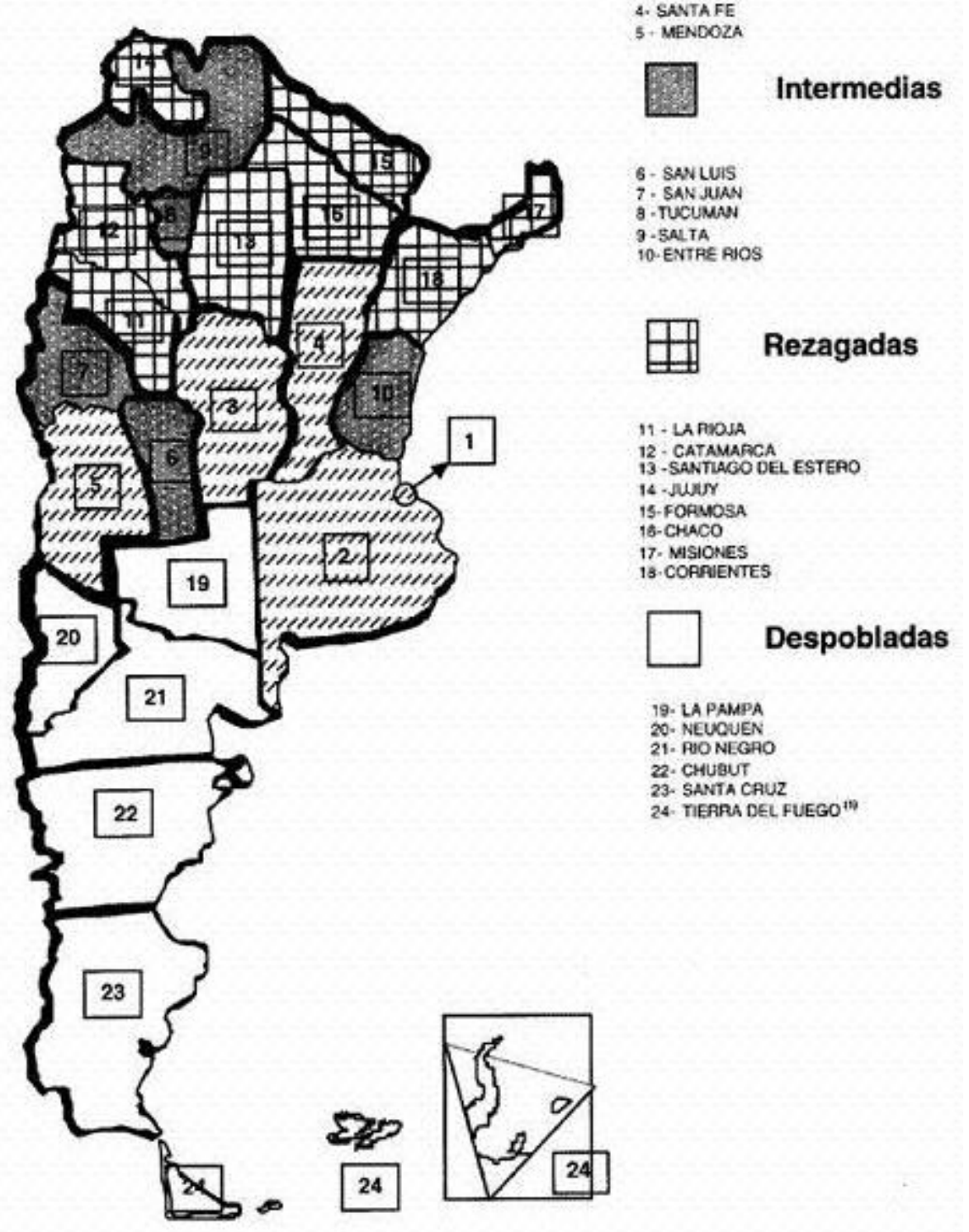

1. GiId Avanzadas

2. BUENOS AIRES

3. CORDOBA

4. SANTA FE

- MENDOZA

ENTAE RIO

- SAMTLGO DEL ESTERO

Jutior

CHACO

7. MSIONES

Despobladas

19.

20. NEUCUEN

21. PUO NEGA

23. SANTA CAUZ

- TERRA DEL FUEGO

Nota: (1) El nombre completo es Provincia de Tierra del Fuego, Antátida e Islas del Attäntico Sur

En la Tabla 2 presentamos los resultados que arrojó la recopilación de datos vinculados con la disponibilidad del gobierno en línea para los ciudadanos, el e-gov como herramienta de relación con contratistas y proveedores, la existencia de normativas y diferentes regulaciones de estímulos a las TICs. 
Tabla 2

Provincias Argentinas - Gobierno Electrónico (Año 2001)

\begin{tabular}{|c|c|c|c|c|}
\hline Jurisdicción & $\begin{array}{c}\text { Disponibilidad } \\
\text { E-gov }\end{array}$ & Normativa & $\begin{array}{l}\text { Contratistas y } \\
\text { proveedores }\end{array}$ & $\begin{array}{l}\text { Estimulos } \\
\text { a las TICs }\end{array}$ \\
\hline Buenos Aires & 3 & 4 & 1 & 1 \\
\hline Ciudad de Bs. As. & 2 & 0 & 0 & 0 \\
\hline Córdoba & 3 & 4 & 1 & 0 \\
\hline Mendoza & 3 & 3 & 1 & 0 \\
\hline Santa Fe & 2 & 0 & 1 & 0 \\
\hline Promedio avanzadas & 2.6 & 2,2 & 0.8 & 0.2 \\
\hline Entre Rios & 1 & 1 & 0 & 0 \\
\hline Salta & 1 & 0 & 0 & 0 \\
\hline San Juan & 2 & 2 & 1 & 0 \\
\hline San Luis & 2 & 4 & 1 & 0 \\
\hline Tucumán & 2 & 3 & 0 & 0 \\
\hline Promedio intermedias & 1.6 & 2,0 & 0.4 & 0.0 \\
\hline Catamarca & 2 & 0 & 0 & 0 \\
\hline Chaco & 2 & 4 & 1 & 0 \\
\hline Corrientes & 0 & 0 & 0 & 0 \\
\hline Formosa & 1 & 0 & 0 & 0 \\
\hline Jujuy & 1 & 1 & 0 & 0 \\
\hline La Rioja & 2 & 4 & 1 & 1 \\
\hline Misiones & 4 & 4 & 1 & 0 \\
\hline Sgo. del Estero & 0 & 3 & 0 & 0 \\
\hline Promedio rezagadas & 1.5 & 2.0 & 0.4 & 0.1 \\
\hline Chubut & 2 & 2 & 0 & 0 \\
\hline La Pampa & 2 & 2 & 0 & 0 \\
\hline Neuquen & 2 & 0 & 0 & 0 \\
\hline Rio Negro & 2 & 4 & 0 & 0 \\
\hline Santa Cruz & 2 & 1 & 1 & 0 \\
\hline Tierra del Fuego & 3 & 2 & 0 & 0 \\
\hline Promedio despobladas & 2,2 & 1,8 & 0,2 & 0,0 \\
\hline Promedio general & 1.9 & 2.0 & 0.4 & 0.1 \\
\hline
\end{tabular}

Fuente: Encuestas a Provincias e investigación en Intemet

En términos generales puede decirse que los resultados guardan un cierto paralelismo con las características de la clasificación de provincias utilizada, en tanto las jurisdicciones avanzadas muestran los índices más altos, seguidas por las despobladas, intermedias y rezagadas. En el caso de estas dos últimas se verifica la inexistencia de diferencias sustanciales.

En términos generales puede decirse que los resultados guardan un cierto paralelismo con las características de la clasificación de provincias utilizada, en tanto las jurisdicciones avanzadas muestran los índices más altos, seguidas por las despobladas, intermedias y 
rezagadas. En el caso de estas dos últimas se verifica la inexistencia de diferencias sustanciales.

Yendo a un análisis de los diferentes ítems analizados, se observa que en el caso de normativas, las provincias intermedias y las rezagadas, se ubican después de las avanzadas quedando mejor posicionadas que las jurisdicciones despobladas.

Otro aspecto a resaltar son los bajos puntajes del indicador "regulaciones y promociones", lo que estaría indicando que a excepción de Buenos Aires y La Rioja ninguna jurisdicción posee sistemas impositivos, entes reguladores, regímenes de promoción sectorial a las empresas y/o de régimen local de incentivo o promoción a la inversión en TICs. Similar análisis le cabe a la dimensión "Contratistas y Proveedores" que arroja en general resultados muy alejados del óptimo, lo que hace pensar en un bajo nivel de difusi ón del gobierno electrónico en el ámbito de compras y contrataciones del Estado.

En la Tabla 3 se presentan los resultados del relevamiento de los sitios web de los tres poderes que conforman el gobierno provincial. Nuevamente, y con mayor énfasis que lo observado en la Tabla 2, se reproduce la lógica de la clasificación de provincias utilizada, ubicándose en primer lugar las jurisdicciones avanzadas, seguidas por las despobladas, luego las intermedia y por último las rezagadas.

Tabla 3

Provincias Argentinas - Sitios web por Poderes (Año 2001)

\begin{tabular}{|c|c|c|c|c|c|c|}
\hline \multirow[t]{2}{*}{ Jurisdiccion } & \multicolumn{2}{|c|}{ P. Elecutivo } & \multicolumn{2}{|l|}{ P. Judicial } & \multicolumn{2}{|l|}{ P. Legislatiwo } \\
\hline & Direcdion & Resultado & Direction & Resultado & Direction & Resultade \\
\hline Buencs Alives & wwwgtagovar & 2 & wweseta sov.af & 5 & $\begin{array}{l}\text { wrw. sentolotagos.ar } \\
\text { www hodiguados-bagovix }\end{array}$ & 5 \\
\hline Cudad de Bs. As. & wow buencedires govir & 2 & masistoaires goviar & 5 & wsw:logstafuragovar & 3 \\
\hline Cordoba & swecbagoviar & 2 & WWw.justiciacondoba.gov.ar & 4 & Wwis.dpitadas condobagor.ar & 4 \\
\hline Mandoza & Ww mendcza gor ar & 3 & 'wnw jus mendoca $.00 \mathrm{var}$ & 3 & www legisaturamendoza.gor ar & 4 \\
\hline SantaFe & wwwsartale gow a & 3 & wWw.podegudicial-se.gov, af & 1 & mow logistaturasartafe.gov at / & 4 \\
\hline Promedio avanzadas & & 2,4 & & 3,6 & & 40 \\
\hline Ertro Rics & wWWentrerics opvar & 2 & & 0 & 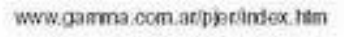 & 0. \\
\hline Sala & www.gcbismosida.gon.af & 0 & wWo Elusidasatragon, ar & 3 & www.ciendessita.gor.ar & 1 \\
\hline San Jean & www.sanguangovar & 1 & wesspbhagoyat & 1 & wow kgistatka-sanjuanosgar & 2 \\
\hline Sancus & www.sanlus.govar & 2 & & 0 & & $\theta$ \\
\hline Tucuman & aww.tucumaniowar & 1 & warjustuctumngovat & 3 & $\begin{array}{l}\text { Wwo usuarike advance.com aritlog. } \\
\text { tuc }\end{array}$ & 3 \\
\hline Promedio intermedias & & 1.2 & & 1,4 & & 12 \\
\hline
\end{tabular}

Sigue $\rightarrow$ 
Tabla 3

Provincias Argentinas - Sitios web por Poderes (Año 2001) (Cont.)

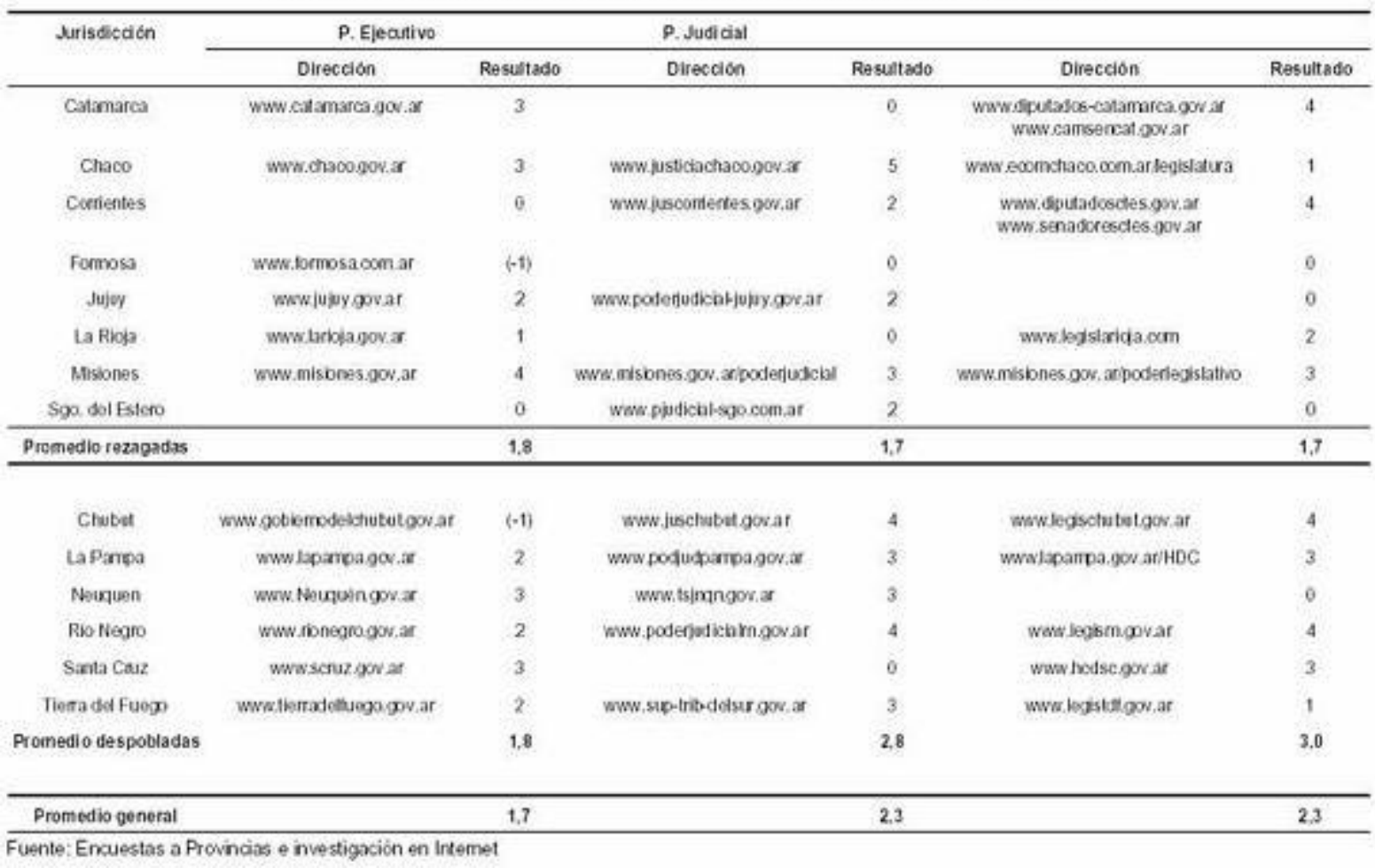

En lo que hace al análisis particular de cada punto, todas las provincias -salvo Santiago del Estero y Corrientes- tienen sitio web del poder Ejecutivo. Sin embargo, el $71 \%$ de los mismos son únicamente informativos (contenidos estáticos) y/o de interacción básica (intercambio de información vía e-mail). En un 40\% de los sitios se publican las licitaciones en marcha pero en ningún caso se observó la posibilidad de tramitarlas electrónicamente. Según el testeo, los niveles de accesibilidad son buenos en el $75 \%$ de los sitios, pero no ocurre lo mismo con la actualización, ya que el $25 \%$ de los sitios son actualizados con una periodización que supera el mes, lo cual estaría indicando una débil vinculación con los procesos administrativos y de gestión cotidianos.

En el $70 \%$ de las jurisdicciones el Poder Legislativo tiene sitio web, el que posee una buena accesibilidad en el $54 \%$ de los casos. No obstante, la mayoría de ellos son meramente informativos y sólo el $17 \%$ incluye compras y contrataciones. En ningún caso se advirtió la posibilidad de seguimiento o de participación on - line en debates, tampoco la posibilidad de la consulta popular electrónica.

En cuanto al Poder Judicial, en el relevamiento se encontraron mejores estándares que los del Legislativo. El $78 \%$ de las jurisdicciones tiene sitio web del poder Judicial con una buena accesibilidad en el $67 \%$ de ellos. Sólo uno de cada cinco permite algún tipo de gestión on - line, la mitad cuenta con base de datos de legislación y jurisprudencia, el 
$15 \%$ tiene registros de abogados y peritos y sólo el $12 \%$ incluye compras y contrataciones.

Sobre los resultados que se han volcado hasta aquí, y con el fin de poder construir una imagen de la distancia de cada jurisdicción con respecto al valor para el total del país, se calculó un registro adicional al que denominamos "brecha" bajo la siguiente fórmula:

Brecha de la Jurisdicción = Valor País / Valor Jurisdicción *100

Se trata de un indicador inverso: cuanto menor es el porcentaje de brecha, mayor es el avance relativo de la provincia en el tema en cuestión.

De manera similar a los guarismos que se presentan en la Tabla 4, los resultados globales correspondientes al desarrollo del gobierno electrónico transcriptos en la Tabla 5 mantienen la estructura general, donde, las provincias avanzadas aparecen como las jurisdicciones mejor posicionadas seguidas por las provincias despobladas. Por último, en la situación de menor desarrollo relativo, encontramos a las provincias intermedias y a las provincias rezagadas.

Tabla 4

Argentina. Cuadro comparativo de algunas variables por regiones

\begin{tabular}{lcccc}
\hline Áreas & Superficie & Población (2001) & PBG (2001) & \% Des. Hum. (2001) (2) \\
\hline Central & 27,2 & 66,9 & 78,8 & 142,4 \\
Despoblada (1) & 33,5 & 5,6 & 7,2 & 119,4 \\
Intermedia & 15,2 & 12,6 & 7,5 & 93,1 \\
Rezagada & 24,2 & 14,9 & 6,4 & 63,3 \\
Total & $100 \%$ & $100 \%$ & $100 \%$ & 100 \\
\hline
\end{tabular}

Nota: (1) No incluye Territorio Antärtico Argentino / (2) Para Promedio simple del pais $=100$ Fuente: Provinfo, 2003 y PNUD (2002).

Sin embargo, dentro de estos dos grupos de provincias el orden está invertido; si bien por un margen estrecho, las provincias rezagadas tienen mayor nivel de desarrollo en egov que las provincias intermedias.

Profundizando este análisis, observamos algunas anomalías que explican porqué las rezagadas han superado a las intermedias: las provincias de Chaco y Misiones presentan guarismos muy desarrollados. En el caso de la última provincia citada, su puntaje la coloca como segunda jurisdicción en el consolidado nacional con 19 puntos sobre 28. 
Otros puntos a destacar son el bajo nivel de desarrollo de dos provincias intermedias (Entre Ríos y Salta) que muestran registros de provincias rezagadas, y el caso de Formosa que presenta resultados que directamente salen de escala.

\section{Conclusiones}

El presente relevamiento se considera el primer intento sistemático de construir un panorama sobre el nivel de avance del Gobierno Electrónico en las 24 jurisdicciones que componen la República Argentina. En este sentido, la posibilidad de haber llevado adelante la tarea y la presentación de la información constituye un importante logro en sí mismo.

Sin pretender agotar el análisis de la importante variedad y cantidad de datos recopilados, se pueden resaltar algunas situaciones que, estimamos, son las conclusiones más interesantes que pueden extraerse de la información presentada en este caso: 
Tabla 5

Argentina. Indicadores de: Disponibilidad del E-gov, normativa, contrataciones, regulaciones y promociones y sitios web de los tres poderes (Año 2001)

\begin{tabular}{|c|c|c|}
\hline Jurisdicción & Totales & Brecha \\
\hline Buenos Aires & 21 & $51 \%$ \\
\hline Ciudad de Bs. As. & 12 & $89 \%$ \\
\hline Córdoba & 18 & $59 \%$ \\
\hline Mendoza & 17 & $63 \%$ \\
\hline Santa $\mathrm{Fe}$ & 11 & $97 \%$ \\
\hline Total avanzadas & 15,8 & $68 \%$ \\
\hline Entre Rios & 4 & $268 \%$ \\
\hline Salta & 5 & $214 \%$ \\
\hline San Juan & 9 & $119 \%$ \\
\hline San Luis & 9 & $119 \%$ \\
\hline Tucumán & 12 & $89 \%$ \\
\hline Total intermedias & 7,8 & $137 \%$ \\
\hline Catamarca & 9 & $119 \%$ \\
\hline Chaco & 16 & $67 \%$ \\
\hline Corrientes & 6 & $178 \%$ \\
\hline Formosa (") & 0 & $1.070 \%$ \\
\hline Jujuy & 6 & $178 \%$ \\
\hline La Rioja & 11 & $97 \%$ \\
\hline Misiones & 19 & $56 \%$ \\
\hline Sgo del Estero & 5 & $214 \%$ \\
\hline Total rezagadas & 9.0 & $118 \%$ \\
\hline Chubut & 11 & $97 \%$ \\
\hline La Pampa & 12 & $89 \%$ \\
\hline Neuquén & 8 & $134 \%$ \\
\hline Rio Negro & 16 & $67 \%$ \\
\hline Santa Cruz & 10 & $107 \%$ \\
\hline Tierra del Fuego & 11 & $97 \%$ \\
\hline Total despobladas & 11,3 & $95 \%$ \\
\hline Promedio general & 10,8 & $100 \%$ \\
\hline
\end{tabular}

Fuente: Encuestas a Provincias e investigación en Intemet.

Nota ("): Como el resultado total tue igual a cero, a los fines de poder calcular la brecha se lo consideró como igual a 0,1

1. En virtud de los diferentes aspectos observados a través de la investigación, se puede afirmar que, como ocurre en casi toda la experiencia internacional, los avances en la gran mayoría de las provincias argentinas se encuentran en una etapa inicial o incipiente del desarrollo del gobierno electrónico, en tanto ofrecen servicios informativos (contenidos estáticos) y/o de interacción básica (intercambio de información vía e-mail). 
2. Reproduciendo el patrón de desigualdad regional, se verifica la existencia de una brecha en el nivel de despliegue de e-gov con un importante grado de paralelismo al de la brecha económico-social. Las provincias avanzadas son las jurisdicciones de mayor desarrollo relativo, seguidas por las provincias despobladas y finalmente el grupo de las jurisdicciones calificadas como intermedias y rezagadas.

3. En el marco de este patrón general existen algunos elementos a destacar. En primer lugar, las provincias que definimos como "despobladas" muestran un cierto avance relativo en comparación con su situación socioeconómica. De acuerdo a una investigación paralela $\underline{6}$, todo parece indicar que este hecho, se origina en las demandas que genera el emprendimiento turístico de importante desarrollo en la región, lo que induce un "avance" general en el desarrollo de las TICs que impacta en el e-gov.

4. Una importante "anomalía" al patrón descrito se observa en nivel de avance del gobierno electrónico en el caso de las provincias rezagadas, el que muestra un grado de desarrollo mayor que el observado en las jurisdicciones intermedias.

5. A priori, puede inferirse que en el caso del e-gov, esta situación se debe a que se trata de una actividad que no estuvo determinada por las fuerzas del mercado, sino que tuvo que ver con otros factores como por ejemplo la voluntad política de llevar adelante un proyecto específico. En este sentido, se destaca el caso de la provincia de Misiones, con un nivel de desarrollo del gobierno electrónico que sería el correspondiente al de una provincia avanzada.

En resumen, estos resultados, si bien confirman a grandes rasgos la hipótesis de equiparación, también muestran que algunas jurisdicciones han podido alcanzar un desarrollo en gobierno electrónico superior al que se deduciría de su desarrollo socioeconómico.

\section{Notas}

* Este trabajo reseña una investigación que fue realizada en el marco del Consejo Federal de Inversiones (CFI) por los autores en conjunto con Ángel Vaca, Ricardo Luque, Sergio Mordacci y Roberto Esteso, y que culminara en noviembre de 2002 .

1. Al respecto ver CFI (2002).

2. Ver clasificación de provincias utilizada. 
3. Una de estas subvariables (accesibilidad) contiene, a su vez, cuatro dimensiones.

4. Metodología para el análisis de la accesibilidad: Se diagnosticaron los 63 sitios web detectados de las 24 jurisdicciones provinciales mediante un testeador automático (Watson Addy), y a través de un chequeo personal de cada uno de ellos para medir la accesibilidad por medio de cuatro variables: 1 . La respuesta del server a la dirección correspondiente: Podía resultar positiva o negativa. 2. Velocidad de acceso a la página: Tiempo en segundos con una conexión dial up de 56 Kbps. 3. Su compatibilidad con los servidores de búsqueda: La que podía ser buena, regular o mala en la medida que tuviera etiquetas (Meta tags) de descripción de la página y de palabras clave que facilitaran ser encontradas por los buscadores (browsers). 4.La popularidad del sitio: Cantidad de páginas que mencionan la página (links). Se diseñaron intervalos diferentes para sitios gubernamentales, legislativos y judiciales y se clasificaron en: de popularidad alta; media y baja. Se valorizaron las respuestas de modo de poder calificar la accesibilidad de cada sitio: 1) Respuesta del server: $\mathrm{Sí}=1 ; \mathrm{No}=0.2$ ) Velocidad: Un segundo $=3$; menos de 10 segundos $=2$; más de 10 segundos $=1.3)$ Compatibilidad: Buena $=3$; regular $=2 ;$ mala $=1.4$ ) Popularidad: Alta $=3$; media $=2$; baja $=1$. Respecto del cuarto punto, se valorizaron las respuestas a la popularidad con escalas diferentes de acuerdo a si el sitio pertenecía al Poder Ejecutivo, Legislativo o Judicial. La escala es la que se muestra en la Tabla 1. Con los cuatro aspectos valorizados como se expuso, los sitios web podían obtener un puntaje máximo de 10 puntos. Finalmente, se obtuvo el puntaje de cada sitio y se le otorgó un punto en accesibilidad a los que tuvieran un puntaje igual o superior a cinco; a los que no alcanzaron ese puntaje se los calificó con cero puntos en este aspecto.

5. Clasificación de Provincias utilizada: Cualquier visión que se haga de la totalidad del país no puede pasar por alto el nivel de heterogeneidad y desigualdad que lo recorre en su dimensión política, social, económica, demográfica, etc. Una importante cantidad de trabajos han abordado el tema, empezando por los clásicos de Núñez Miñana (1972) y Romero y Rofman (1997, edición original 1973). Posteriormente se desarrollaron otras clasificaciones, como las de Esteso y Capraro (1989) y Cao y Rubins (1994), que incorporaron a sus marcos teóricos los acontecimientos ocurridos a partir de la desestructuración del Estado Interventor, sin llegar a resultados sustancialmente diferentes. En el presente estudio se trabaja con la clasificación que utiliza en sus publicaciones sobre provincias Argentinas el Banco Mundial (ver por ejemplo Banco Mundial 1996 y 1998), la que divide al país en cuatro categorías: Avanzadas o centrales: Se caracterizan por ser las jurisdicciones que presentan una concentración de población y un aparato productivo de dimensiones muy superiores al que se observa en otras áreas del territorio. De ésta manera, constituyen a escala nacional, el sector más dinámico en la evolución de la estructura económica y social. I ntermedias: Se trata de aquellas provincias que, sin compartir el tamaño ni el dinamismo de las provincias avanzadas, han generado productos que se insertan en las provincias del área central, presentando ritmos de desarrollo económico y social intermedios. Rezagadas: Son las provincias que no lograron desarrollar actividades de exportación hacia el área central y que exhiben una extendida presencia de formas de producción no capitalistas. Durante buena parte del último siglo y medio fueron el punto de partida de migraciones hacia el área central y en términos relativos, en estas provincias se encuentra el menor nivel de ingreso per-cápita y los índices de mayor deterioro social. Despobladas: Ubicadas al sur del país se caracterizan por la baja densidad poblacional relativa, fruto del tardío proceso de ocupación 
y desplazamiento de la población aborigen (fines del Siglo XIX). Dentro de su aparato productivo se destaca la existencia de algunos emprendimientos muy desarrollados como el petrolero y el turístico, conviviendo con explotaciones ganaderas extensivas. Algunos indicadores transcriptos en la Tabla 3 dan cuenta de las notables diferencias que existen entre las diferentes áreas que integran el territorio.

6. Al respecto ver Vaca (2002).

\section{Referencias Bibliográficas}

1. Banco Mundial (1996), Argentina provincial finances study. Selected issues in fiscal federalism. Banco Mundial Report: 15487 AR, Washington, EEUU.

2. Banco Mundial (1998), Provincial finances. Update '98, Banco Mundial, LCSPR, Washington, EEUU.

3. Cao, Horacio y Rubins, Roxana (1994), "La estructura institucional de las provincias rezagadas". En: Realidad Económica № 128, IADE, Buenos Aires.

4. CFI - Consejo Federal de Inversiones (2002), Las Nuevas Tecnologías de Comunicación e Información y las Administraciones Públicas Provinciales - CFI Argentina - disponible en: www.cfired.org.ar/esp2/indices/f_biblio.htm Consulta: $15 / 03 / 03$.

5. Dahl, Robert (1983), Análisis político actual. EUDEBA (Editorial Universitaria de Buenos Aires), Buenos Aires.

6. Esteso, Roberto y Héctor Capraro (1989), "Algunos elementos para el análisis de las relaciones entre el Estado Federal y las Provincias", en Cuadernos IIPAS № 1, Fundación Friedrich Ebert, Buenos Aires.

7. Núñez Miñana, Horacio (1972), Indicadores de Desarrollo Regional en la República Argentina: Resultados Preliminares, Documento Interno № 10. Facultad de Ciencias Económicas, UNLP, La Plata, Buenos Aires.

8. ONU (2003), América Latina en el mundo. Situación de América Latina y el Caribe en cuanto a la capacidad de Gobierno Electrónico (E-Gov capacity). Programa de Administración Pública de las Naciones Unidas y American Society for Public Administration, tomado de: www.top.org.ar/ Boletin/BoletínN3.htm Consulta: 01/05/03. 
9. PNUD (2002), Programa de las Naciones Unidas para el Desarrollo. Aportes para el desarrollo humano de la Argentina / 2002. Un enfoque integral, Buenos Aires, Argentina.

10. Provlnfo (2003), Base de Datos de la Unidad de Información Integrada, Ministerio del Interior, tomado de: www.mininterior.gov.ar/provinfo/bd_provinfo/base /index3.htm Consulta: 15/05/03

11. Rofman, Alejandro y Romero, Luis (1997), Sistema socioeconómico y estructura regional en la Argentina, Amorrortu Editores, Buenos Aires (Edición original 1973).

12. Vaca, Josefina (2002), “Las Tecnologías de la Información y la Comunicación en la Argentina: Un enfoque regional". En: Revista Venezolana de Gerencia, Año 7 № 17, Universidad de Zulia, Venezuela.

13. World Markets Reserch (2001), Global e-Goverment Survey. World Markets Reserch, EE.UU. 